\title{
EL “CASO CÉSPEDES” DE CABELLO/CARCELLER Y LA AUTOBIOGRAFÍA TRANS
}

\author{
Rafael Manuel Mérida ${ }^{1}$ \\ [Reseña de: Cabello/Carceller, Anexo: Caso Céspedes']
}

Este catálogo, en edición bilingüe (español e inglés), fue publicado con motivo de la exposición Cabello/Carceller. Borrador para una exposición sin título (cap. III), que se mostró en Ciudad de México, entre el 9 de febrero y el 26 de mayo de 2019, en el Museo Universitario de Arte Contemporáneo de la UNAM. La muestra, como sugiere su título, supuso la continuidad de un proyecto cuyo segundo “capítulo" pudo contemplarse en el Centro de Arte Dos de Mayo, en Madrid, entre enero y mayo de 2017, que bien debe considerarse una retrospectiva de su trayectoria. ${ }^{3}$ Tanto el proyecto artístico, comisariado en ambos casos por Manuel Segade, como la propuesta historiográfica de reevaluación de una genealogía (auto)biográfica trans del catálogo me parecen de sumo interés.

La pareja formada por Cabello/Carceller es un equipo que posee una dilatada trayectoria en el panorama artístico español e internacional, según confirman muy diversas exposiciones "individuales" y colectivas. Formado en 1992 por Helena Cabello (1963) y Ana Carceller (1964), constituye un referente en el ámbito de las artes de las últimas décadas y, en concreto, en el de la performance, el video y la instalación; a mi juicio, Cabello/Carceller son ineludibles para conocer el desarrollo de las prácticas artísticas feministas, lésbicas y queer en el Estado español, según sugería también Juan Vicente Aliaga (2013). Además de su actividad creativa, ejercen la docencia en la Facultad de Bellas Artes de la Universidad de Castilla-La Mancha, en Cuenca. Como se indica en su sitio de internet, uno de sus objetivos sería el cuestionamiento de "las narrativas modernistas que ignoran a las minorías políticas mientras fingen recurrentemente acudir a ellas”, de tal manera que

\footnotetext{
${ }^{1}$ Profesor Serra Húnter de literatura española y de estudios de género (Facultad de Letras, Universitat de Lleida. España).

2Este catálogo puede descargarse gratuitamente desde el sitio de internet de "Acción Cultural Española": https://www.accioncultural.es/es/cabello/carceller-anexo-caso-cespedesebook.

${ }^{3}$ https://ca2m.org/es/historico/item/2525-cabello-carceller.
} 
potencian la interrogación sobre "los modos de representación hegemónicos en las prácticas visuales" e intensifican las "alternativas críticas". ${ }^{4}$

La que(e)rencia del equipo por la personalidad de Elena/o de Céspedes - en torno a la cual y a partir de la cual se desarrolló la exposición mexicana - no es nueva. Basta con recordar A/O (Caso Céspedes), exhibida en el Centro Andaluz de Arte Contemporáneo (Sevilla, 2010) y en A Present Without Memory: A/O (Céspedes Case), en la Magill Library (Haverford College, Filadelfia, 2016), que son sus precedentes. El atractivo de este personaje histórico, que vivió en la Andalucía de la segunda mitad del siglo $\mathrm{XVI}$, es consonante con las preocupaciones éticas y políticas del equipo en torno a las identidades de género. Así, en una conferencia dictada en Valencia en el año 2000, ya reflexionaba sobre "la experiencia real de haber nacido y de habitar en sociedades que todavía no se encuentran preparadas para aceptar a aquellas y aquellos que saltamos por encima de las barreras conocidas" (Cabello y Carceller, 2001, p. 137-138).

El catálogo se abre con una síntesis de Segade sobre el emplazamiento de este proyecto inacabado en el pensamiento y la producción de Cabello/Carceller, así como sobre las significaciones que potencia el diálogo entre pasado y presente a partir de Elena/o:

Nació en Granada, mujer, mulata y esclava, pero llegó a ser cirujano y se casó por la iglesia como hombre con una mujer, para ser luego juzgada y condenada por la Inquisición española. En el video que realizan Cabello/Carceller, un personaje de género indeterminado, Alex, investiga los jardines y estancias del Monasterio de la Cartuja de Sevilla, el edificio barroco donde se sitúa actualmente el Centro Andaluz de Arte Contemporáneo. Allí, Alex busca localizaciones para una narrativa sobre Céspedes, deconstruyendo el espacio de exhibición como espacio de producción, del mismo modo que la propia exposición de su trabajo contribuye a la dislocación de los lazos convencionales que fijan la representación a la identidad (SEGADE, 2019, p. 9).

${ }^{4}$ http://www.cabellocarceller.info/cast/index.php?/info/. 
La segunda y tercera sección del catálogo son aportaciones del hispanista estadounidense Israel Burshatin, de quien conocemos sus diversos estudios sobre Céspedes (por ejemplo, BURSHATIN, 1999). Mientras que la tercera sería un "Resumen de eventos notables en la vida de Eleno de Céspedes" (p. 59-61), la segunda sección, y más reveladora (titulada "Obras invisibles: visualidad, contemplación y verdad en $A / O$ (Caso Céspedes, p. 2941), ofrece en primera instancia una síntesis de la biografía de quien ha podido considerarse desde uno de los mejores testimonios de hermafroditismo en la España de la Contrarreforma hasta un caso eximio de picaresca por parte de una persona que sumaba toda suerte de subalternidades: sexo, género, raza, religión, grupo social, ... No obstante, a continuación, reflexiona sobre el video exhibido:

En la película A/O (Caso Céspedes), las artistas Cabello/Carceller sugieren un enfoque alternativo a la problemática de cómo leer y conectarse con un disidente queer cuyas experiencias de vida, mentalidad e idiomas eran bastante diferentes a los nuestros. Y, sin embargo, no podemos no quedar hipnotizados y conmovidos por la aparición de hilos comunes que atraviesan vidas queer hoy día y en la Iberia de la modernidad temprana (BURSHATIN, 2019, p. 36).

La transcripción de una selección de fragmentos del proceso de Eleno de Céspedes en el Tribunal de la Inquisición de Toledo, en 1587-1589, preparada por Burshatin y Cabello/Carceller, resulta tan elocuente como deslumbrante (p. 66-109) y se antoja la imprescindible antesala del guion de las artistas de su video, con voz narrativa en off, que se ofrece en las p. 157-160. De este guion y de esta voz podemos destacar algunas frases, como las siguientes:

El resumen que aparece en la portada de las actas reduce a unas pocas líneas el significado de toda una vida de trasgresión. El juicio y la defensa del orden imperante configuran un relato que rozaría la literatura fantástica... si no fuera trágico. Nos queda el recuerdo porque algunos actos considerados criminales fueron meticulosamente registrados en los archivos. [...] ¿Dónde se quedó Céspedes? ¿En qué parte del camino decidió que debería saltar de un lado al otro, o en qué parte del camino los 
demás decidieron que no podía vivir en medio? [...] Individuo a individuo, poco a poco, estudiando cada caso y destruyendo uno a uno al diferente se naturalizó el artificio. Eso nos demuestran los archivos y este será el tema central de la película. Juicio a juicio, condena a condena, acoso tras acoso... Introduciendo en nuestras mentes la autocensura, golpeando y humillando a cada niña y niño disidentes [...] (CABELLO/ CARCELLER, 2019, p. 157-159).

Según sugería Burshatin (2019, p. 40), el relato del siglo XVI contendría para Álex, protagonista del video, una promesa de su íntima genealogía a través de "una temporalidad queer, fusionando el presente (al explorar los jardines) y el pasado (la narrativa de Eleno)". Esta sería la apuesta de Cabello/Carceller, que, simultáneamente, estaría mostrando el documento histórico (las actas del juicio inquisitorial) y transformándolo, iluminándolo y velándolo. O sencillamente ampliando y actualizando las significaciones de una trayectoria que ha solido entenderse desde una concepción binaria de los sexos (véanse Maganto Pavón, 2007, y Ruiz Rodríguez - Hernández Delgado, 2017), mucho más ajena a los discursos sobre el género de la Europa de los siglos XVI y XVII de cuanto debiéramos, según certifica, a modo de ejemplo, otra personalidad histórica tan atractiva como fuera Catalina de Erauso, la “monja alférez", en muy parecido contexto (CLEMINSON/VÁZQUEZ GARCÍA leminson, 2013) ${ }^{5}$.

Estaríamos de acuerdo con Vázquez García (2018, p. 15), cuando afirma que "[d]esignar con el término 'transexual' a individuos del siglo XVI (como la célebre morisca granadina Helena de Céspedes) o del primer tercio del siglo XX (como el pintor danés Einar Wegener, convertido en Lili Elbe), que cambiaron de sexo, implica por tanto incurrir en un anacronismo". Sin embargo, considero que este anacronismo - que debe evitarse en la investigación académica - posee un enorme potencial artístico, como el presente volumen confirma. Por otra parte, en pleno siglo XXI, la construcción de una nueva modalidad de autobiografía trans, y de su

${ }^{5}$ Tengamos presente, por otra parte, que tanto la biografía de Céspedes como la de Erauso han podido ser interpretadas en el contexto del homoerotismo lésbico de aquellos siglos: véase Velasco (2003). 
genealogía, bien pudiera y debiera escapar de las ortodoxias morales y retóricas impuestas durante centurias por dogmas religiosos y científicos. ${ }^{6}$ MÉRIDA, Rafael Manuel. Reseña de Cabello/Carceller, Anexo: Caso Céspedes,

Ciudad de México: RM Verlag, 2019. ISBN: 978-84-17047-84-9.

\section{Referências}

ALIAGA, Juan Vicente. Apuntes para una cartografía de la "homosexualidad" en el arte en el Estado español (1970-1995). In: Minorías sexuales en España (1970-1995). Textos y representaciones. Ed. R. M. Mérida. Barcelona: Icaria, 2013, p. 47-65.

BURSHATIN, Israel. Written on the Body: Slave or Hermaphrodite in Sixteenth-Century Spain. In: Queer Iberia. Sexualities, Cultures, and Crossings from the Middle Ages to the Renaissance. Ed. J. Blackmore-G. S. Hutcheson. Durham: Duke University, 1999, p. 420-456.

BURSHATIN, Israel. Obras invisibles: visualidad, contemplación y verdad en A/O (Caso Céspedes). In: Cabello/Carceller, Anexo: Caso Céspedes. Ciudad de México: RM Verlag, 2019, p. 29-41.

BURSHATIN, Israel. Resumen de eventos notables en la vida de Eleno de Céspedes. In: Cabello/Carceller, Anexo: Caso Céspedes. Ciudad de México: RM Verlag, 2019, p. 59-61.

CABELLO, Helena-Ana CARCELLER. ¡Yo no soy nadie! ¿Quién eres tú Representaciones de ciertas androginias inclasificables en el arte contemporáneo. In: Miradas sobre la sexualidad en el arte y la literatura del siglo XX en Francia y España. Ed. J. V. Aliaga. Valencia: Universitat de València, 2001, p. 125-138.

\footnotetext{
${ }^{6}$ Este trabajo forma parte del proyecto de investigación "Memorias de las masculinidades disidentes en España e Hispanoamérica" (PID2019-106083GB-100) del Ministerio de Ciencia e Innovación del Gobierno España.
} 
CABELLO / CARCELLER. A/O (Caso Céspedes). Guion voz narradora. In: Cabello/Carceller, Anexo: Caso Céspedes. Ciudad de México: RM Verlag, 2019, p. $157-160$.

CLEMINSON, Richard-Francisco VÁZQUEZ GARCÍA. Sex, Identity and Hermaphrodites in Iberia, 1500-180o. Londres: Pickering \& Chatto, 2013.

MAGANTO PAVÓN, Emilio. El proceso inquisitorial contra Elena/o de Céspedes (1587-88): Biografía de una cirujana transexual del siglo XVI. Madrid: Método Gráfico, 2007.

RUIZ RODRÍGUEZ, Ignacio-Alexander HERNÁNDEZ DELGADO. Elena o Eleno de Céspedes. Un hombre atrapado en el cuerpo de una mujer en la España de Felipe II. Madrid: Dykinson, 2017.

SEGADE, Manuel. La cesura entre los cuerpos. In: Cabello/Carceller, Anexo: Caso Céspedes. Ciudad de México: RM Verlag, 2019, p. 8-27.

VÁZQUEZ GARCÍA, Francisco. La invención del sujeto transexual. In: En todos los colores. Cartografías del género y las sexualidades en Hispanoamérica. Ed. Mo C. Bianciotti-Ma N. González-D. Fernández Matos. Barranquilla: Universidad Simón Bolívar, 2018, p. 13-34.

VELASCO, Sherry. Interracial Lesbian Erotics in Early Modern Spain: Catalina de Erauso and Elena/o de Céspedes. In: Tortilleras. Hispanic and U.S. Latina Lesbian Expression. Ed. L. Torres - I. Pertusa. Filadelfia: Temple University, 2003, p. 213-227.

Recebido em 18 julho de 2020.

Aceito em 30 de outubro de 2020. 